\title{
Working from home is the new black: into the private world of remote collaboration in COVID-19 lockdowns
}

ARNAUD PROUZEAU, Inria Bordeaux, France and Monash University, Australia

JOANNE MIHELCIC, Monash University, Australia

LONNI BESANÇON, Monash University, Australia

To mitigate the impact of the COVID-19 pandemic, countries around the globe implemented Non-Pharmaceutical Interventions (NPIs), one of which being Working From Home (WFH). In this paper we present an ethnographic investigation into the adaptations of working spaces and habits due to the adoption of WFH. We interviewed 12 participants from different industry contexts in order to cover a wide range of tools and practices used to conduct remote work. We focus on the importance and benefits of the different technologies available and how they impact collaboration. We discuss challenges experienced by participants in organizing their workspace at home, the impact of workload on practices, and the growing worries about isolation. The findings highlight the importance of understanding the changing physical, social and technological environments in designing new ways of working and collaborating remotely. From our results, we finally derive new directions for the HCI and CSCW research agenda on the topic of WFH.

Additional Key Words and Phrases: Working from Home, Remote collaboration, Ethnography

\section{INTRODUCTION}

To slow the rapid spread of COVID-19, in March 2020 a large number of countries decided to implement lockdowns which restricted their population's movement and led a large number of people to work from home full-time. Whilst working from home (WFH) is not a new concept [16], COVID-19 and the sudden lockdowns across the globe have irrevocably changed the way we conduct business and collaborate with colleagues or clients. In April 2020, the International Labor Organization (ILO) estimated that up to 35\% of the workforce was working from home, compared to around 3\% in the months before [17]. COVID-19 lockdowns forced the distribution of organisational operations into homes of employees in which the only connection between people and the business was technological. It provoked an accelerated rate of digital transformation which crossed well beyond the boundaries of business and industry into the homes of business owners, workers and students, to name a few. But how do people experience and manage the social interactions with their colleagues, in formal contexts, like meetings, as well as in informal ones?

In this paper, we present a study conducted during the first six months of the COVID-19 lockdown in 2020. We investigated the adaptation of hybrid and fully remote meetings or collaborations, to these new working conditions. We focused on the tools and technologies people used for remote collaboration: on their characteristics and affordances and in particular how their use was influenced by prior organisational culture, practices and task related activities.

As we, the researchers, were also constrained to working and therefore conducting research from home, digital ethnography was the methodology of choice [25]. We interviewed 12 participants, across different industry contexts, who were, at the time, working from home, either full or part time. We used video-conferencing tools (in our case Zoom) to conduct and record the interviews, and Miro (https://miro.com/) for collaborative note-taking during the interviews and to assist with data analysis. While such adaptation and persistent changes to the way people work have been predicted by organisational psychologists [26], there is no study, to our knowledge, that explored this with an ethnographic perspective.

Our results suggested that, overall, people managed to do their work while working from home. To do so, however, most participants had to adapt their homes, their working methods, and their daily interaction with their coworkers. 
While this cost was acceptable by participants in this specific context of crisis, most did not see this situation as acceptable in a post-COVID future. Some companies, to assist their employees in this transition, adapted their processes and their tools to this specific situation. For instance, instant messaging tools were setup to limit the number of virtual meetings and keep a social life similar to the one at the office. Such decisions, overall, led to increased contentment. On the other hand, some just replaced face to face meetings with virtual ones using video-conference tools leading to employees feeling more tired and isolated. Overall, this experience suggests that, while research demonstrated that working from home is technically feasible, more research is needed to adapt it to make it as comfortable and as rich regarding social interactions as working in an office when the situation demand it or in a mixed model.

\section{RELATED WORK}

Our work pertains to working from home and its implications in general but also during the COVID-19 pandemic, we thus survey these two aspects below.

\subsection{Working From Home pre-pandemic: heavily studied yet barely adopted.}

Working From Home is not a new concept [16] and there have been many studies on its impact before the COVID-19 pandemic. Early research into the concept laid out the complexity of the topic and its study because of its multifactorial nature[30]: it is difficult to completely isolate the effect of telework because of the impact of family-interactions and overall well-being on one's mental health and performances. Early research in Human-Computer Interaction [32] looked at the importance of providing video-conferencing solutions when compared to audio only and concluded that video was beneficial in some contexts. In a 2004 study with 45 employees [9], Crosbie et al. reported that working from home can be a double-edged sword (see also Tietze [31]) and that further studies and models on the efficiency of working from home should be conducted to model more realistically the work/home context beyond a perfect safe haven. In a study of call-center employees in China in 2014 [6], Working From Home was found to increase satisfaction with work as well as performance and working time. The increase in performance was slightly independent from the increase in working hours. Overall, it seems that available evidence concurs in the increased productivity and potential for improved morale of employees who are working from home [1,18]. Nonetheless, it would seem that most of these gains (and their effect size) depends largely on how remote work is implemented [18] and this was, prior to the pandemic, the main focus of research of most studies [18].

However, past research demonstrates that working from home has been considered an option on only an occasionalto-regular basis for part of the workforce [29]. It is therefore unclear how those findings would hold in a context that would see (almost) all employees working from home on a daily-basis.

\subsection{Working From Home during the pandemic}

To mitigate the impact of COVID-19 and lower transmission rate, many countries globally have decided, while waiting for vaccines or treatments, to implement Non-Pharmaceutical Interventions (NPIs). Lockdowns have manifested in: school closures, stay-at-home orders and remote work. Consequently, in many countries and when possible, employees were all invited or forced to work from home [17]. As such drastic implementations have been unusual, the impact they would have on employees, productivity, or work-life was unclear [2,26]. But some research set out to investigate and report on the exact consequences of this implementation of working from home.

Barrero et al. [4], based on a survey of 30,000 Americans hypothesize that working from home is likely to stay after the pandemic ends for its advantages beyond the working context and its increased productivity, such as reduced 
Working from home is the new black

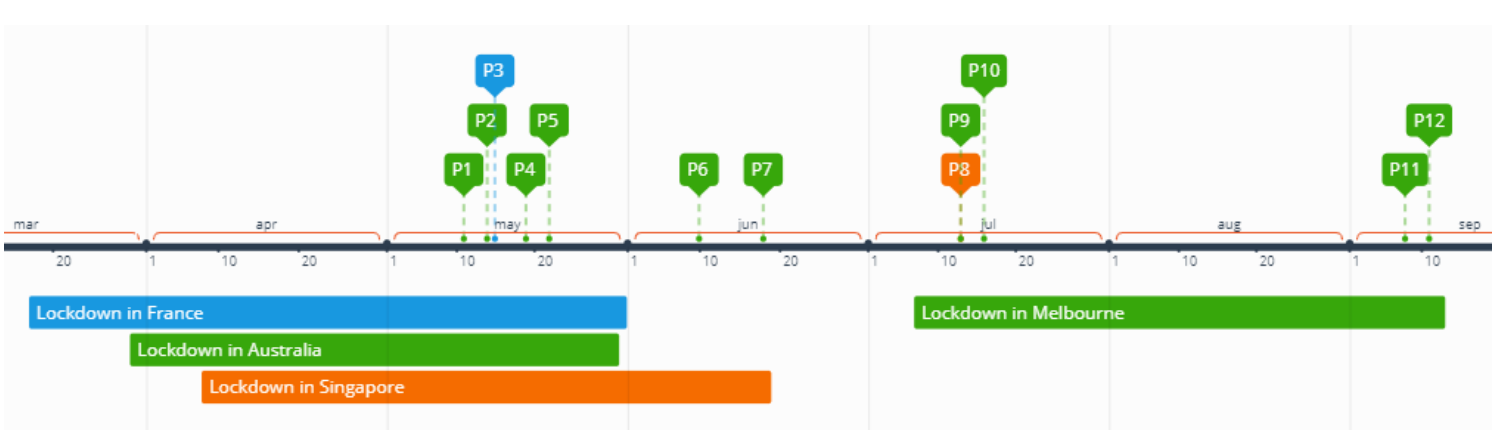

Fig. 1. Timeline with the interviews at the top and the lockdown measures at the bottom. The color encodes the country where the participant lives, or concerned by the lockdown: Australia (Green), France (Blue), and Singapore (Orange).

spendings in expensive city centers or less commuting time. Some research papers also investigated how to make the most of working-from-home from an organizational perspective (e.g., [13]). However, working from home during the pandemic is associated with other new measures (e.g., school closures) that were likely to worsen the psychological risks often correlating with unstructured working time [7]. Similarly, the management of work-life balance of workers during the pandemic depends on the situation and physical space for remote work available at home [3]. Most of these surveys tried to quantify people's responses, performances, or well-being while adapting to compulsory working from home. While such research outcomes and measures are crucial, we were more interested in understanding how people's use of technology evolved to adapt to the situation in different context in order to gain insights on future HCI and $\mathrm{CSCW}$ challenges that our community should focus on.

\section{STUDY DESIGN}

We conducted an ethnographic study on how people experience working from home in the COVID-19 crisis in 2020. More specifically, we focused on the social and technological aspects of working from home, on how people experience and manage the social interactions with their colleagues, in formal contexts, like meetings, as well as in informal ones. There are already several studies on working from home, or teleworking. However, we were interested in the particular circumstances of this period in which a large number of workers (and entire companies) were forced to transition to remote work in a very short time. The use of an ethnographic approach was then justified as we wanted to stay open to themes not predefined for our analysis and to ground our observation in the specific context of the COVID-19 crisis [5].

\subsection{Context}

The COVID-19 disease, caused by the coronavirus SARS-CoV-2, was first identified in the city of Wuhan in China in December 2019. Highly infectious, the disease had spread to the 5 continents by March 2020 and has been declared as a pandemic in the same month by the World Health Association (WHO) [37]. To limit this spread, most countries implemented more of less strict stay-at-home order, or lockdown, and by mid-april, more than 3 billions of people were living under such orders [27]. At the same time, according to the ILO, around 59 countries implemented working from home as a requirement for non-essential workers [17]. 


\subsection{Participants}

We recruited 12 participants via word of mouth (7 females and 5 males). Each had a different occupation at the time of the interview. While our list is far from exhaustive, we tried to have participants with a variation of occupations in function of the type of collaboration (Software engineers mostly collaborate with their teammates when salesman mostly with clients), and the type of information on which they collaborate (Architects collaborate on visual information, like maps or 3D models, when recruitment officers focus more on textual information). It was important for us to include managers in our set of participants, as this position traditionally requires a regular collaboration with the team. All participants have been working from home permanently for at least a month without interruption at the time of the interview (see Figure 1). Thus, we can consider that they were out of the adaptation period for this new context.

Ten of our participants were working in Australia at the time of their interview (see all details in Table 1). Four were interviewed during the first lockdown, which lasted from the 29th of March to the 31st of May (the end date is specific to Victoria, where the 4 participants worked). Two were interviewed after the end of the first lockdown. However, as encouraged by the local government, most companies did not open their office, and the two participants were still working from home at this period. Four participants were interviewed during the second lockdown, which only concerned the city of Melbourne (where the 4 participants worked) and lasted from the 7th of July to the 26th of October. The participants were working from home since the beginning of the first lockdown, except P11, who went back to the office between the two lockdowns and partially during the second one as he was working on projects considered essential. One participant was working in France and he was interviewed during the first lockdown which lasted from the 17th of March to the 2nd of June. Finally, 1 participant worked in Singapore and was interviewed after the first lockdown in Singapore, but was working from home as it was encouraged by the local government.

\subsection{Procedure}

All our interviews were done via video-conference to respect the COVID-19 sanitary measures. Before the interview, we send an explanatory statement and a consent form to the participants by e-mail. We asked them to send us a picture of their physical working space before the interview. Two researchers were present during each interview and lead the interview in an informal manner. We always started with questions about their current position and then asked them to describe the picture they sent us. Following the ethnographic interview methodology, the rest of the interview was not scripted. When one of the author was asking the questions, the other was taking notes on an interactive whiteboard (using Miro ${ }^{1}$ ), on which was pasted the picture sent by the participant before the interview. The link to the whiteboard was shared to the participants and they were allowed to look at it during the interview and even interact with it. Only P2 interacted with the board to draw on the picture. In addition to the notes, the interview were recorded and automatically transcribed (using Otter.io ${ }^{2}$ ). The transcriptions were then checked and corrected by one of the author. We limited the interview to 1 hour to avoid fatigue for the participants (who, for most of them, do several video-conference meetings per day).

\subsection{Analysis methodology}

We performed a Thematic Analysis following Braun and Clarke's methodology [8]. There are a few reasons to explain this choice: (1) we do not intend to discover universal truth as every companies are different and WFH was implemented in different ways (in function of the country, the company policy, the sector). Even inside a company, employees

\footnotetext{
${ }^{1}$ https://miro.com/
}

${ }^{2}$ https://otter.ai/ 
Table 1. Summary table of the participants, with their occupation, the location they were working in at the time of the interview and its timing (i.e. if they were in lockdown or not at the time of the interview).

\begin{tabular}{|c|c|c|c|}
\hline ID & Occupation & Location & Timing \\
\hline P1 & Recruitment officer at university and student & Australia & \multirow{5}{*}{ During lockdown 1} \\
\hline $\mathrm{P} 2$ & Software engineer & Australia & \\
\hline P3 & IT consultant & France & \\
\hline $\mathrm{P} 4$ & Civil servant in government & Australia & \\
\hline P5 & University library manager & Australia & \\
\hline P6 & Urban planner & Australia & \multirow{3}{*}{ After lockdown 1} \\
\hline P7 & Architect & Australia & \\
\hline $\mathrm{P} 8$ & Aircraft salesman & Singapore & \\
\hline P9 & Associate Professor & Australia & \multirow{4}{*}{ During lockdown 2} \\
\hline P10 & Project manager and instructor & Australia & \\
\hline P11 & System integration engineer & Australia & \\
\hline P12 & IT manager & Australia & \\
\hline
\end{tabular}

implemented WFH differently. (2) Our purpose was to identify interesting (and potentially rare) events, so we did not want to just count occurrences. To this end, we took an inductive and iterative approach to derive themes purely from data. The coding was performed by an HCI researcher and a sociotechnical researcher. The analysis of the first two interviews was performed collaboratively by the two researchers using both the miro board, the video and the transcript. These sessions helped to create a first mind map with the different themes (that were not fixed). The other interviews were analysed by one researcher who also filled the mind map. The mind map and the themes were reviewed frequently by both researchers.

\section{RESULTS}

Here we present the result of our thematic analysis by describing the different themes and expand with specific situations that come directly from the interviews.

\subsection{WFH Organisation}

In this section, we report about how the different participants organised their work at home.

4.1.1 WFH before COVID-19. WFH was not a new concept at the beginning of the COVID-19 crisis, it was actually done already by a few participants, not on a full time basis and more or less regularly. P2 and P3 mentioned doing it between one and two days a week, while P10 used to do it around once a month. These days could be used when the participant needed to stay at home for personal reasons (e.g. delivery, specific worker coming at home), or to focus on a specific task ( $\mathrm{P} 2$ mentioned that there can be a lot of interactions/interruptions in the open spaces at work and staying at home allows to isolate themselves).

However, most companies did not permit it for more than a limited number of days, and required people to have a good ergonomic installation at home, as P6 explained, in order to make sure that the employees have a good working setup. This ergonomic checkup has been abandoned in most companies (due to the short time to transition), only P12 mentioned that employees still had to go through official validation of their setup from the occupational safety and health department. Only one participant, P3, experimented a full WFH period before COVID, because of a large public transportation strike in Paris in 2019. 
4.1.2 Transition to full WFH. The implementation of WFH with the first wave of COVID-19 (March-April 2020) happened almost overnight. In addition, most countries (including Australia, France and Singapore) implemented stay-at-home measures (i.e., lockdowns) which restricted people local movements and closed their borders, which restricted more global movements. These impacted various sectors of the economy, and thus, many companies could not have the same level of activity. One of the most hit sector was air travel (more than $70 \%$ of the passengers flights were cancelled in April-May 2020), which impacted P8. P1 and P5 both worked in a university administration and also saw their activities quite reduced due to the loss of many international students for Australian universities (a revenue loss estimated at 3 billions of dollars in 2020).

This lack of activities led to people having fewer meetings with their colleagues and thus, less contact. This lack of connectivity was highlighted by almost half of the participant during the interviews, well illustrated by P6: "I miss the camaraderie with colleagues". This led to two main phenomenons: (1) some people felt more and more isolated, to a point where "people start disappearing" (P6); and (2) the communication inside the company is negatively impacted, leading to difficulties in asking for help or even understanding what is happening in the company. P10 and P4 saw their team being restructured at the beginning of the lockdown, and the lack of information made it more stressful than it already was. P12 also explained that starting new projects was complicated during this period.

On the other hand, some companies just saw minor impacts on their activities. For the participants working in such companies, most of what used to be physical meetings had been replaced by virtual ones using tools such as Zoom or Teams. Some participants (like P7, P9 and P10) spend hours on such tools per day, which was found to be very tiring (this phenomenon would be called "Zoom fatigue" by the media). As already observed in previous studies about WFH $[2,11]$, the boundary between their office and their home started to be blurred, both physically as your home IS your office, P9 actually mentioned they "I miss having my house as my house"; and also temporally as "everyone know you are not far from the office" (P7), and thus you should be able to answer to an email at any time. To avoid such line-blurring, discipline is necessary to create new routine and make sure to stop working at a specific time (P3).

Still, it remains that the experience was not overall negative for the participants. Many explained that there were benefits in working from home: you had a chance to be more focused and thus get more things done (P4, P6, P7, P9), because you do not commute, you can wake up later or do other things around the house (P10). P8 who experienced a high decrease of their activity, reported having more social calls with their team and planning meetings to exchange knowledge regarding specific topic that would not happen prior to the pandemic.

4.1.3 The "home" in Working From Home. Regarding the physical working space, we saw a large spectrum of conditions. From the participants who could have a separate room with a very ergonomic office (most of the time a spare bedroom) like P4, P5, and P7, to participants who shared the dinner table with their partner in the living room like P3, P8, and P9. Most participants were happy about the room itself (after all, it is from their own house), about the fact that they have windows and natural light (which is not the case in all the regular offices), P1 actually mentioned that "being next to a window makes me less tired". On the other hand, some had to work in the same room as their partner, which provoked disturbances such as concurrent video calls (P3, P9), with P3 having to go to the bedroom for long call, or even the fire alarm occurring in the middle of a meeting due to the other cooking (P9). In some cases, the table used as a desk was the dinner table, which led to P6 having to eat with monitors around, or P9 explaining that they do not eat on the table during the week (they removed everything on Fridays). This variety of location as an office led to video meeting with people on their bed, in inappropriate situations (e.g., without a tee shirt on, or drinking a glass of wine), as experienced by $\mathrm{P} 10$. 
For the office itself, most participants could borrow their material from the office (external monitors, chair) to create a comfortable setup (P1, P4, P5, P6, P7, and P9). For the webcam, most people used their laptop; 4 participants actually just opened their laptop to use the webcam (P1, P5, P6, P7). Regarding the headset, except for P8, it was not provided by the companies, and they had to buy it themselves. For the internet connection, people had to rely on their own connection, and some upgraded theirs (paid by the company, or deducted from taxes). Participant were, in general, positive regarding their setup. Although some missed their office setup (P1, P2, P10) since it was more ergonomic.

\subsection{Collaboration during COVID-19}

In this section, we dive specifically on the topic of collaboration and how people managed to work together while working from home.

4.2.1 Tools put in places. Participants mentioned several tools that were used to collaborate with either their coworkers or external partners. Some were already in use when they started working from home (e.g. e-mails), but other were quite new and introduced after (e.g. Instant messaging). In general, all participants used e-mails and a video-conference tool, but instant messaging were only mentioned by half of the participants.

E-mails are still used a lot to collaborate with others. In the case where the company setup an instant messaging tool, e-mails were still used to collaborate with external people (P10). It was also used to get formal feedback regarding documents (P6, P8, P9). When e-mails were also the main tool to communicate internally, it was found to be a bit cumbersome (P4, P6) and less flexible (P10); some participants, like P11 tried to avoid them.

Instant messaging tools, like Slack or Discord, have been setup in some companies before the lockdown, and some started using them after it. They are mostly used to have synchronous communications, but also can be used in an asynchronous way. They seen as mostly informal, and thus, very efficient: "no need for please or thank you" (P2). P2, who works in an IT company reported using them for probably $95 \%$ of their communication. As we will see, it is also used as a social function (P6), however, because of the ease to talk with someone, it can be a bit too much, and few participants complained of the overload of notifications they received because of it (P2, P7).

We will expand on video-conference tools in a later section, but they were reported as preferable for talks about complex topics (P2, P5) or when working closely with a colleague: "it's like working on the same desk" (P3). Thanks to the sharing screen functionalities (P3) or virtual whiteboard (P7, P10), it is possible to provide context about what people are talking about, similarly to a face to face meeting. However, compared to face to face, participants still lacked facial expressions, body language, and conversations did not seem very natural as it is not possible talk over each other (mostly due to technical constraints, for instance, Zoom always look for the primary speaker, as it was reminded by P7).

4.2.2 Small talks. In general, small talks between colleagues is one of the main missing elements in WFH and is considered as important by participants for several reasons. It is necessary (1) to create connection between people (P4, P5); (2) to learn internal processes of the company (P6); (3) to keep up to date with general information (especially as P6 explained: "when things are going crappy" in the team because of restructuring); (4) as a manager, it is very important to catch up with staff (P5). It used to happen, in the office, when you ran into someone in the hallway, at the water cooler/coffee machine, or even, as P3 explained, at the beginning or end of a meeting.

Participants found different solutions to replace these casual small talks, by organising for instance one to one meeting with their colleagues (P5). In this case, they needed to make an explicit effort to organise it (they were afraid of disturbing if they had called without warning first). For example, P5, a manager, said they had to plan the call to her employees in their calendar and find, sometime, a random reason for the meeting in order to catch up with everyone 
and make sure they were going well. Instant messaging (like Teams or Slack) was also used: some companies organised specific channels for informal conversations (P6, P7). Finally, P7 described that they sometime used the chat channel of Zoom to have side conversations during meetings as small talks, it was as if "whispering into someone's ear".

Some participants found some benefits in this lack of small talks. It led to less gossip and in general meant that "you don't have to make an effort now to socially talk to someone" (P6).

4.2.3 Interruption. Interruption, when an employee "disturbs" another employee to have a chat or just ask a question (about a specific process for instance), is quite frequent in an office and important in complex and dynamic environments. To interrupt someone in the office does require a bit of effort both physically, as you have to stand up and walk, and socially, as you have to disturb someone when they are focused on a task.

When working from home, digital tools can be easily used to interrupt a coworker without much effort. This has a positive effect as it encourage people to do it to ask for help for instance (P6). On the other hand, both P2 and P7 described that it felt like everyone is available at any time, and that people were less cautious about who should be connected to a specific topic, which could lead to too many interruptions (lack of etiquette on how to manage this in WFH), and an overload of notifications. P2 mentioned that it led them to deactivate notifications from the instant messaging tool (in this case, Slack) when they want to focus. But to work, such strategy has to be accepted by everyone at the office (else, one would expect a quick answer if they interrupt you).

4.2.4 Social events. Social events in the company happened before the pandemic, but it was not the norm. The lack of social life during lockdown led companies to increase the number of social events. Several participants (P3, P6, P7, P8) reported having virtual weekly drinks, mostly on Friday after work, afternoon tea and coffee break, and even some more advanced social events like quizzes or cocktail-of-the-month party (P7).

These events tended to be more frequent than social event in "real life" (P3) and the audience seemed to be broader (P7 - due to the fact that it was easier to attend) which had a positive impact. Video-conference tools were used to "spice" the event, like breaking rooms in which people are put in randomly (P6). The impact of such events were mostly positive, but not as much as real social events, from which they were a pale ersatz. The fact that video-conference tools do not reproduce a real face to face conversation was exacerbated as explained by P7: "you have to stick with one conversation at a time, conversation does not flow and you cannot hear everyone laugh when you make a joke."

Such events were mostly done in companies with permanent staff, P10, who is a contractor said that they were were most of the time ignored: "there is less of a care factor". WFH increase the gap between the different types of contract.

4.2.5 Technical training. Participants did not report having any specific training regarding the use of the different collaborative tools. When they were already familiar with them because there were already in use before the lockdown, they did not really had any trouble with them, as P6 explained, it actually improved their comfort with the tools. But when the tools were introduced with the lockdown, it led to a few technical issues (P4, P7, P10), and at the beginning "people didn't know how to turn on camera and mic" (P7).

This lack of formal training meant in the first place that the tools were not used at their full potential (and sometime not at all). In times like these, when most of the communication is performed with such tools, it meant that it became harder for employees to be proactive with their work, to ask for assistance, or to mentor other co-workers (P4).

4.2.6 Cyber-security. While cyber security was not the primary focus of this study, organisations are increasingly concerned with the security of data and technology and the risk that this poses on a number of levels [23]. Participants described some of the assumptions they made about cyber security and some of the physical challenges in managing 
technology for working virtually. Regarding the importance of cyber security when working virtually and online, only a small number of participants explained that as the computers were configured and managed by their organisation they did not feel it was necessary to implement further caution in relation to cyber security - that the organisation was responsible for cyber security as a technical issue (P2, P3, P8). Participants also articulated that the technology and especially internet access was not always reliable especially when working from home. This sometimes impacted on their ability to access shared and secure drives for working with or storing data and information - which are critical assets for any organisation (P3). Finally, P5 described issues or concerns when meetings or collaborating with people external to the organisation. Providing 'visitor' access to online systems created an added risk to security when these 'visitors' as the host organisation could not always control the technology and tools being used to access these systems.

\subsection{The specific case of virtual meetings}

Whilst collaborative technologies were already being used within organisations, these tools were described as being adopted more extensively to facilitate collaboration and communications which were, prior to COVID-19, being conducted face-to-face.

Most participants were already familiar with remote meetings, WFH just changed the amount of remote meetings they had to do. It also meant that everyone was remote, which made everyone more "equal" (P3 reported that the remote participants before were most of the time less engaged).

4.3.1 Organisation. In addition to their usual meetings, most companies organised either daily or weekly catch-ups with the team (P2, P3, P4, P5, P6, P7, P9, P10, P11). This could have several benefits: (1) keeping everyone in touch, and (2) getting updates on the different projects. This would be usually at the beginning of the day/week, and would last between 15 and 30 minutes.

In addition to being a threat to their work-life balance, as explained earlier, some participants mentioned that being never far from the office also meant that it was harder to avoid a meeting (P7, P10), even outside of their work hours. More generally, both P7 and P10 explained that it was harder to protect their time in their calendar, meaning that if another co-worker saw a gap, they would not hesitate to plan a meeting. The number of meetings also increased as it is not possible to just walk to another co-worker desk to have a quick chat, "even a 5 minutes meeting needs to be planned" (P11).

On the other hand, meetings became more flexible. It is easy to do something else at the same time; as P7 stated, "you are one click away from the mall". Drop-in and drop-out are more acceptable. And because of the removal of the geographical constraints, it was easier to have every necessary people in a meeting. Few participants noticed that in general meetings were shorter. While this does not seem to be a general rules, however, they seem to stick with the allocated time slot (P1, P12).

The biggest issue met by participants during their meetings would be the sound quality. Quickly people invested in good mic and headset (as P7 stated, not having it is a bit like "showing up without a pencil"), and also to mute themselves when they are not speaking (P2, P7). To mute someone else is actually considered as acceptable (which would probably not be in face to face).

4.3.2 Tools. The tools the most used for virtual meeting were Zoom (P1, P2, P9) or Teams (P4, P8), and sometimes both at the same time (P3, P5, P6, P7, P10, P11, P12). In the latter case, participants tended to use one for more informal communication (between co-workers, or for social events), and one for more formal meeting. P1 mentioned using Hangout at the beginning (mostly due to licence limitation for Zoom), but it was considered as less professional. Zoom 
was considered by some participants to have a better video layout (P6, P10), as "you can cram 20 people on the screen" (P6).

The chat was considered in general as a useful tool during virtual meeting (P1, P4, P6, P7). It was mainly used to create order, to comment or give side notes during a talk/class. P7 considered it could represent a way to have side conversation (which would be equivalent to "whispering into someone's ear").

Another good functionality from the video-conference tool was the possibility for one user to share their screen (P1, P3, P4, P5, P6, P10). This functionality was mostly used to imitate meeting at someone's desk and showing something on the screen, or talking over a presentation. Overall, participants considered it was better than nothing, and managed to work on different types of documents with this functionality: code, presentation slides, maps, architect drawings. Using the "whiteboard" functionalities, other participants could even draw on it (which was easier than describing something). P6, as an urban planner, used it to share a snippet of a map and draw on it.

Finally, The different tools offered useful functionalities that imitate real life situation, like "Raising hand" which allows students to ask a question in class (P1). There is also the breakout room to separate the participants in a virtual meeting into different rooms, mostly used in class or for social events (P6, P10).

4.3.3 Use of the video. The video is one of the main functionalities of video-conference tools. Overall, every participant though that the video is important for different reasons: for the non-verbal communication and to read body language (especially true for non-native speakers - P5, P7), to show engagement (P3, P10), to show presence (P7), to gives a better office atmosphere (P4, P7, P12), to show leadership in management position (P9). P12 went even further by explaining it helps making the meeting more real.

However, participants also considered that the video is one of the main factor of Zoom fatigue: it sends a lot of information that are hard to integrate (everyone on the screen, where do they look, etc. - P7, P9, P10), Some people do not like to see themselves (P5), There can be kids in the background (P7). Having the video on during working from home can be seen as an intrusion into someone's home (P5 explained: "You don't want people to see your laundry"), More technically, there can be internet bandwidth issues (P7, P10, P12).

To handle these issues, participants managed the use of their video in different ways: some deactivated it completely (P5). This is an issue as most of the video-conferencing software (e.g. Zoom) are programmed to show in priority people with video on the screen. So someone who does not turn it on can "disappear". P4 and P12 reported that such a behaviour could be interpreted as a sign of isolation and a need to be reached out to. Some deactivate under certain circumstances, like when they are not really involved in the meeting (P7). P9 reported that even if they know that they do not have to have the video, they will keep it as a sign of leadership. They admitted that once, they had to cut because of a technical problem, and really enjoyed it.

Regarding the intrusion of people in their home, one solution have been to use Virtual Background (mostly popularized by Zoom). While people are still using them, it can be seen as a more fun functionality and will not be used in a more formal context (P5, P6, P7). People really worked on "Background staging" (P6), meaning arrange their background to be "clean" or use a virtual background that looks like a real house.

\subsection{WFH in the future}

In the future, most participants wish to see a mixed model, they like the flexibility of being able to work from home, and this experience proved that "you do not need to be in the office to get things done" (P6) and that "it is possible to operate without anyone in the same room" (P7). However, being physically present in the office is important, especially when 
you start a new job (P2), and in general, participants wanted to get back human contact and to get out of home during the day (P2, P3, P6). This solution was actually already in place for P11 who was able to spend some time at the office during the lockdown, they found it pretty good, even if the days at the office ended up being very long as they tried to do as much as they could there.

A mixed model seems, on the paper, a good solution. However, as P9 mentionned, this means that you would need to have a work setup both at the office and at home (which is not the case now as lots of workers took their office materials). During meetings, people working from home could be at a disadvantage as they would be on a screen only compared to the others physically present. P7 proposed to solve this by having everyone participate from an ipad/screen, even if they are colocated, and we could not require people to specify if they are going to be on site or not. It is also important to remember that not everyone can WFH in good conditions (P12), so having an office for everyone is still very important.

\section{DISCUSSION AND CONCLUSION}

Overall, our ethnographic study highlighted the complex nature of working from home and its potential benefits and risks found before (e.g., [9, 31]) or during the pandemic (e.g., [13, 26]) Participants of our study highlighted many benefits of WFH echoing previous large surveys conducted during the pandemic [4]: workers can save on commuting time and be more focused for instance. They also reflected on difficulties highlighted by previous work in particular when it comes to work-life balance and physical organization or one's home for remote work [3]. It seems that overall, our participants also envision that working from home is here to stay [4], although careful considerations should be given to when and how to do it (see subsection 4.4).

This study also highlights the fact that we should not just try to work in the same way, just with virtual tools. For instance, a virtual meeting using a video-conference tool is more tiring and less engaging than a face to face one and thus, we should not just interchange them but find new processes and new methods better adapted to this context. Our ethnographic approach allows us to shed light on interesting solutions proposed by either companies and the employees themselves, and understand how tools were put in place. A recurring example was the use of instant messaging tools (e.g. Slack or Discord) to recreate office interactions that used to happen for instance at the coffee machine (e.g. small talks). This created, in return, new issues (like an increase of interruptions from co-workers) that should be investigated. To avoid having employees isolated, and keep a mentor-mentee dynamic, to give formal technical training on these new tools is key.

\subsection{Limitations}

Our study of workers during the pandemic naturally exhibits some limitations. The first one lies in our rather small number of participants. However, considering that we conducted and transcribed face-to-face interview, this number appears reasonable when compared to previous ethnographic research in HCI (e.g., [19]). The second and probably main limitation of our work is in the fact that the people we interviewed were all from different backgrounds, industry contexts, or countries. However, this choice was made in order to discover a wide and diverse set of issues that we believe would be less likely to emerge with a more homogeneous group of participants. This fits very well with our goal to try and identify new challenges and implications of the pandemic and working from home on HCI research. We detail these below. 


\subsection{Implication for $\mathrm{HCI}$ research}

We now describe how the findings from our ethnographic study can reveal new interesting area of future work or highlight the need to consider a wider spectrum of research outcome in HCI research.

First, as our results suggest, WFH is a viable options for companies, and what the COVID-19 crisis demonstrated that it was possible to very quickly transition to a full remote operation in many sectors. It is important to note that this transition was largely done thanks to employees adapting their way of working, their personal space and in general, their personal life. Thus, while it can be considered as a positive experience, it cannot be considered as a long term solution. Our study suggests that the participants that has the most positive experience of WFH were actually the participants working in companies that adapted their process and their tools to the specific context of remote work. The massive use of instant messaging tools which played a social function as well as replaced some of the face to face collaboration was a good example. On the contrary, participants in companies that only replaced face to face meeting with visio conference felt overall tired and isolated. While we do not think that WFH should be the norm, we believe that it will be part of the workplace of the future. Both the HCI and CSCW community should explore how to adapt the process and the tools to provide a working experience at home that would be as rich as in the office.

Another particular interest to the HCI community lies in the tools that participants and companies have used to meet or maintain social relationships and small talks with colleagues during the pandemic. According to our participants, social events with coworker were more accessible but video-conferencing tools failed to reproduce the fluid interactions of real-world events. While virtual reality setups could help mitigate some of these issues [20] (e.g., VR conferencing [10] or social VR [15]), our data stresses that the wide adoption of the technology it would require is unlikely to happen soon since companies rarely provide hardware beyond a simple laptop. Our data further reflects on past work investigating how people use and segregate between messaging applications and the level of intimacy they have with the people they contact [24]. For instance it would seem that some of our participants used different applications for their formal video-calls than they used for their informal ones. How or why they make such a distinction would be an interesting avenue for future work. Similarly, we believe that studying people's use of emojis (e.g., [14, 35]) in the specific context of work with the massive adoption of work messaging apps would be an interesting investigation.

Finally, much of past work on video-conferencing focuses their research measures/outcomes on the feeling of presence or immersion which has been found to be improved with immersive headset experiences (augmented or virtual reality) $[28,33]$ and to positively influence performance [36]. On the one hand, VR/AR headsets could help reduce the number of monitors hardware needed to work from home and therefore help workers save space and configuration time [20]. On the other hand, it seems unlikely that all office work, even the most immersive one, could be done without a physical screen or additional input devices (see, e.g., [34]). We therefore believe that our findings help highlight that future work on collaborative work and technologies should consider the challenges of working from home and would add to already identified challenges regarding collaboration using immersive technologies [12]. The adoption of immersive technologies does not solely rely on their prices or their performances solely anymore, but also on how they could be adopted at home for remote workers depending on their price, their potential to invade workers' privacy $[21,22]$, or the possibility for workers to arrange and the time it might take to prepare [21] their workspace at home to use them.

Our findings highlight the importance of considering the $2020 \mathrm{WFH}$ period as the first large experiment of transition to teleworking. We believe our contribution will directly benefit the HCI community as it gives, through our findings, 
incentives to dive into this period to understand how people managed this specific context, and to adapt its research agendas with a community-wide discussion.

\section{AUTHORS CONTRIBUTION}

Arnaud Prouzeau: conceptualization, investigation, data curation, project administration, writing - original draft, writing - review \& editing.

Joanne Mihelcic: conceptualization, methodology, investigation, data curation

Lonni Besançon: writing of the original draft, writing - review \& editing.

\section{REFERENCES}

[1] Zara Abrams. 2019. The future of remote work. Monitor on Psychology 50, 9 (Oct. 2019). http://www.apa.org/monitor/2019/10/cover-remote-work

[2] Tammy D. Allen, Timothy D. Golden, and Kristen M. Shockley. 2015. How Effective Is Telecommuting? Assessing the Status of Our Scientific Findings. Psychological Science in the Public Interest 16, 2 (2015), 40-68. https://doi.org/10.1177/1529100615593273 arXiv:https://doi.org/10.1177/1529100615593273 PMID: 26403188.

[3] Tammy D Allen, Kelsey Merlo, Roxanne C Lawrence, Jeremiah Slutsky, and Cheryl E Gray. 2021. Boundary management and work-nonwork balance while working from home. Applied Psychology 70, 1 (2021), 60-84. https://doi.org/10.1111/apps.12300

[4] Jose Maria Barrero, Nicholas Bloom, and Steven J Davis. 2021. Why Working from Home Will Stick. Working Paper 28731. National Bureau of Economic Research. https://doi.org/10.3386/w28731

[5] Isabelle Baszander, Nicolas Dodier, and David Silverman. 2004. Ethnography: Relating the part to the whole. SAGE, London, 9-34. https: //contentstore.cla.co.uk//secure/link?id=eb4686dd-884a-e611-80bd-0cc47a6bddeb

[6] Nicholas Bloom, James Liang, John Roberts, and Zhichun Jenny Ying. 2014. Does Working from Home Work? Evidence from a Chinese Experiment *. The Quarterly fournal of Economics 130, 1 (11 2014), 165-218. https://doi.org/10.1093/qje/qju032 arXiv:https://academic.oup.com/qje/articlepdf/130/1/165/30629971/qju032.pdf

[7] Hanifa Bouziri, David RM Smith, Alexis Descatha, William Dab, and Kévin Jean. 2020. Working from home in the time of covid-19: how to best preserve occupational health? Occupational and environmental medicine 77, 7 (2020), 509-510. https://doi.org/10.1136/oemed-2020-106599

[8] Virginia Braun and Victoria Clarke. 2006. Using thematic analysis in psychology. Qualitative Research in Psychology 3, 2 (2006), 77-101. https: //doi.org/10.1191/1478088706qp063oa arXiv:https://www.tandfonline.com/doi/pdf/10.1191/1478088706qp063oa

[9] Tracey Crosbie and Jeanne Moore. 2004. Work-life balance and working from home. Social Policy and Society 3, 3 (2004), $223-233$. https: //doi.org/10.1017/S1474746404001733

[10] Sylvie Dijkstra-Soudarissanane, Karim El Assal, Simon Gunkel, Frank ter Haar, Rick Hindriks, Jan Willem Kleinrouweler, and Omar Niamut. 2019. Multi-Sensor Capture and Network Processing for Virtual Reality Conferencing. Association for Computing Machinery, New York, NY, USA, $316-319$. https://doi.org/10.1145/3304109.3323838

[11] Kimberly A. Eddleston and Jay Mulki. 2017. Toward Understanding Remote Workers' Management of Work-Family Boundaries: The Complexity of Workplace Embeddedness. Group \& Organization Management 42, 3 (2017), 346-387. https://doi.org/10.1177/1059601115619548 arXiv:https://doi.org/10.1177/1059601115619548

[12] Barrett Ens, Benjamin Bach, Maxime Cordeil, Ulrich Engelke, Marcos Serrano, Wesley Willett, Arnaud Prouzeau, Christoph Anthes, Wolfgang Büschel, Cody Dunne, Tim Dwyer, Jens Grubert, Jason H. Haga, Nurit Kirshenbaum, Dylan Kobayashi, Tica Lin, Monsurat Olaosebikan, Fabian Pointecker, David Saffo, Nazmus Saquib, Dieter Schmalstieg, Danielle Albers Szafir, Matt Whitlock, and Yalong Yang. 2021. Grand Challenges in Immersive Analytics. Association for Computing Machinery, New York, NY, USA. https://doi.org/10.1145/3411764.3446866

[13] Jennifer Feitosa and Eduardo Salas. 2020. Today's virtual teams: Adapting lessons learned to the pandemic context. Organizational dynamics 32836509 (June 2020), 100777-100777. https://doi.org/10.1016/j.orgdyn.2020.100777

[14] Carla F. Griggio, Midas Nouwens, Joanna McGrenere, and Wendy E. Mackay. 2019. Augmenting Couples' Communication with <i>Lifelines $</ i>$ : Shared Timelines of Mixed Contextual Information. Association for Computing Machinery, New York, NY, USA, 1-13. https://doi.org/10.1145/3290605.3300853

[15] Simon Gunkel, Hans Stokking, Martin Prins, Omar Niamut, Ernestasia Siahaan, and Pablo Cesar. 2018. Experiencing Virtual Reality Together: Social VR Use Case Study. In Proceedings of the 2018 ACM International Conference on Interactive Experiences for TV and Online Video (SEOUL, Republic of Korea) (TVX '18). Association for Computing Machinery, New York, NY, USA, 233-238. https://doi.org/10.1145/3210825.3213566

[16] Penny Gurstein. 1991. Working at Home and Living at Home: Emerging Scenarios. Fournal of Architectural and Planning Research 8, 2 (1991), 164-180. http://www.jstor.org/stable/43029030

[17] International Labor Organisation. 2020. Working from Home: Estimating the worldwide potential. Technical Report. https://www.ilo.org/global/ topics/non-standard-employment/publications/WCMS_743447/lang--en/index.htm

[18] Kaila S. Jacoby and Samantha Holland. 2019. Digital Nomads: The Final Frontier of Work Arrangements? The Bridge: Connecting Science and Practice 56, 3 (Jan. 2019). http://siop.enoah.com/tip/jan18/editor/ArtMID/13745/ArticleID/693/CategoryID/97/CategoryName/563/The-Bridge-Connecting- 
Science-and-Practice-Digital-Nomads-The-Final-Frontier-of-Work-Arrangements

[19] Vera Khovanskaya, Phoebe Sengers, Melissa Mazmanian, and Charles Darrah. 2017. Reworking the Gaps between Design and Ethnography. Association for Computing Machinery, New York, NY, USA, 5373-5385. https://doi.org/10.1145/3025453.3026051

[20] Pascal Knierim and Albrecht Schmidt. 2020. The Virtual Office of the Future: Are Centralized Workplaces Obsolete? (2020).

[21] Kiron Lebeck, Kimberly Ruth, Tadayoshi Kohno, and Franziska Roesner. 2018. Towards Security and Privacy for Multi-user Augmented Reality: Foundations with End Users. In 2018 IEEE Symposium on Security and Privacy (SP). 392-408. https://doi.org/10.1109/SP.2018.00051

[22] Divine Maloney, Samaneh Zamanifard, and Guo Freeman. 2020. Anonymity vs. Familiarity: Self-Disclosure and Privacy in Social Virtual Reality. In 26th ACM Symposium on Virtual Reality Software and Technology (Virtual Event, Canada) (VRST'20). Association for Computing Machinery, New York, NY, USA, Article 25, 9 pages. https://doi.org/10.1145/3385956.3418967

[23] Cedric Nabe. [n. d.]. Impact of COVID-19 on Cybersecurity. https://www2.deloitte.com/ch/en/pages/risk/articles/impact-covid-cybersecurity.html. Accessed: 2021-09-08.

[24] Midas Nouwens, Carla F. Griggio, and Wendy E. Mackay. 2017. "WhatsApp is for Family; Messenger is for Friends": Communication Places in App Ecosystems. Association for Computing Machinery, New York, NY, USA, 727-735. https://doi.org/10.1145/3025453.3025484

[25] Sarah Pink, Heather Horst, John Postill, Larissa Hjorth, Tania Lewis, and Jo Tacchi. 2016. Digital ethnography. Springer. 161-165 pages.

[26] Cort Rudolph, Blake Allan, Malissa Clark, Guido Hertel, Andreas Hirschi, Florian Kunze, Kristen Shockley, Mindy Shoss, Sabine Sonnentag, and Hannes Zacher. 2020. Pandemics: Implications for Research and Practice in Industrial and Organizational Psychology. (May 2020). https: //doi.org/10.31234/osf.io/k8us2

[27] Alasdair Sandford. 2020. Coronavirus: Half of humanity now on lockdown as 90 countries call for confinement. Accessed on the 3rd of December 2020. https://www.euronews.com/2020/04/02/coronavirus-in-europe-spain-s-death-toll-hits-10-000-after-record-950-new-deaths-in-24-hou.

[28] Mickael Sereno, Xiyao Wang, Lonni Besançon, Michael J Mcguffin, and Tobias Isenberg. 2021. Collaborative Work in Augmented Reality: A Survey. IEEE Transactions on Visualization and Computer Graphics (2021). https://doi.org/10.1109/TVCG.2020.3032761

[29] Kristen Shockley. 2014. Telecommunting. techreport. Society for Industrial and Organizational Psychology. http://www.siop.org/Portals/84/docs/ WhitePapers/ScientificAffairs/telecommuting.pdf

[30] Peter Standen, Kevin Daniels, and David Lamond. 1999. The home as a workplace: Work-family interaction and psychological well-being in telework. Journal of occupational health psychology 4, 4 (1999), 368. https://doi.org/10.1037/1076-8998.4.4.368

[31] Suzanne Tietze. 2002. When" work" comes" home": Coping strategies of teleworkers and their families. Fournal of Business Ethics (2002), 385-396. https://doi.org/10.1023/A:1021236426657

[32] Elizabeth S. Veinott, Judith Olson, Gary M. Olson, and Xiaolan Fu. 1999. Video Helps Remote Work: Speakers Who Need to Negotiate Common Ground Benefit from Seeing Each Other. In Proceedings of the SIGCHI Conference on Human Factors in Computing Systems (Pittsburgh, Pennsylvania, USA) (CHI '99). Association for Computing Machinery, New York, NY, USA, 302-309. https://doi.org/10.1145/302979.303067

[33] Cheng Yao Wang, Mose Sakashita, Upol Ehsan, Jingjin Li, and Andrea Stevenson Won. 2020. Again, Together: Socially Reliving Virtual Reality Experiences When Separated. Association for Computing Machinery, New York, NY, USA, 1-12. https://doi.org/10.1145/3313831.3376642

[34] Xiyao Wang, David Rousseau, Lonni Besançon, Mickael Sereno, Mehdi Ammi, and Tobias Isenberg. 2020. Towards an Understanding of Augmented Reality Extensions for Existing 3D Data Analysis Tools. In CHI '20 - 38th SIGCHI conference on Human Factors in computing systems. Honolulu, United States. https://doi.org/10.1145/3313831.3376657

[35] Sarah Wiseman and Sandy J. J. Gould. 2018. Repurposing Emoji for Personalised Communication: Why Pizza Means "I Love You". Association for Computing Machinery, New York, NY, USA, 1-10. https://doi.org/10.1145/3173574.3173726

[36] Bob G. Witmer and Michael J. Singer. 1998. Measuring Presence in Virtual Environments: A Presence Questionnaire. Presence: Teleoperators and Virtual Environments 7, 3 (06 1998), 225-240. https://doi.org/10.1162/105474698565686 arXiv:https://direct.mit.edu/pvar/articlepdf/7/3/225/1836425/105474698565686.pdf

[37] World Health Organisation (WHO). 2020. Timeline: WHO's COVID-19 response. Accessed on the 3rd of December 2020. https://www.who.int/ emergencies/diseases/novel-coronavirus-2019/interactive-timeline. 